\title{
ORIGINAL
}

\section{EDUCAÇÃO EM SAÚDE E NUTRIÇÃO EM JOÃO PESSOA, PARÁ́BA}

\author{
HEALTH AND NUTRITION EDUCATION IN JOÃO PESSOA, PARAÍBA, BRAZIL
}

\author{
Roberto Teixeira LIMA' \\ Jefferson Carneiro de BARROS ${ }^{2}$ \\ Marcos Roberto Andrade de MELO2 \\ Melquisedek Galdino de SOUSA ${ }^{2}$
}

\begin{abstract}
RESUMO
Este estudo analisa as características das práticas de Educação em Saúde e Nutricão no município de João Pessoa, Paraíba, Brasil. Foram investigados 65 Serviços de Saúde, dos 70 catalogados, por meio de visitas e entrevistas com os profissionais para obtenção de informações sobre as práticas de Educação em Saúde. Dos Serviços investigados, 74,0\% desenvolvem a prática de Educação em Saúde. Os profissionais médicos, enfermeiros, psicólogos, odontólogos e assistentes sociais estão presentes na maioria dos Serviços, e apenas 43,7\% dos Serviços têm nutricionistas em seus quadros. Na atuação direta em Educação em Saúde, as assistentes sociais, enfermeiras e psicólogas foram as que apresentaram maior participação. Palestras, cartazes e folhetos foram os principais recursos identificados nas atividades educativas na maioria dos Serviços. A população alvo das atividades foi representada por gestantes, crianças pré-escolares e casais. Dos temas educativos, a maioria dos Serviços trabalha com aleitamento materno, pré-natal, planejamento familiar, higiene pessoal e vacinação. Foi verificado que os Serviços não apresentam uma forma objetiva e sistemática de avaliação das práticas educativas, muito embora a maioria deles considere o retorno dos pacientes aos serviços como forma de avaliação.
\end{abstract}

Termos de indexação: educação em saúde, serviços de saúde comunitária, nutricionista.

\begin{abstract}
This report analyzes characteristics of Health Education practices in the city of João Pessoa, Paraiba State, Brazil. The data were obtained from 65 Health Services of the 70 catalogued, including different health centers, by means of visits and interviews with the health professionals in the Services. Among the investigated Services, $74.0 \%$ practices Health Education. Only $43.7 \%$ of the investigated Services have nutritionists; the most frequent professionals are: doctors, nurses, psychologists, dentists and social workers. In the educational activities, social workers, nurses and psychologists were the health professionals with more participation. For those activities, the most frequent strategies adopted were lectures, posters and pamphlets. Those educacional activities were elaborated for pregnant women, preschool children and couples. It was observed that breast feeding, prenatal care, family planning, personal hygiene and vaccination were the educational themes more discussed in the Services. It was also observed that the Services do not have a systematic form of evaluation of the activities. Most of the Services consider the follow-up of the patient as an index to evaluate the activities.
\end{abstract}

Index terms: health education, community health services, nutritionist.

\footnotetext{
(1) Departamento de Nutrição, Núcleo de Estudos em Saúde Coletiva, Universidade Federal da Paraíba, Av. Augusto F. Maia, 206, Campus I, Cidade Universitária, 58059-900, João Pessoa, PB, Brasil. Correspondência para/Correspondence to: R.T. LIMA. E-mail: teixeira@funape.ufpb.br

(2) Curso de Graduação em Nutrição, Universidade Federal da Paraíba.
} 


\section{INTRODUÇÃO}

Educação em Saúde, entendida como processo, visa capacitar os indivíduos a agir conscientemente diante da realidade cotidiana, com aproveitamento de experiências anteriores, formais e informais, tendo sempre em vista a integração, continuidade, democratização do conhecimento e o progresso no âmbito social. Visa também a autocapacitação dos vários grupos sociais para lidar com problemas fundamentais da vida, tais como nutrição, desenvolvimento biopsicológico, reprodução, tudo isso no contexto de uma sociedade dinâmica (Bezerra, 1986; Valente, 1986; Lavinsky, 1988; Pitta, 1994; Freitas, 1997; Vasconcelos, 1997).

Diante das diversas abordagens pedagógicas existentes no processo de ensino-aprendizagem, nosso grupo optou pela abordagem humanística, segundo a qual a educação tem sentido ampliado e está representada por tudo o que favorece o crescimento pessoal, interpessoal e intergrupal. É nesta abordagem que vemos a Educação em Saúde, em particular a Educação Nutricional, na medida em que o profissional de saúde, enquanto educador que é, deixa seu modo tradicional de educar, para ocupar um papel de facilitador do processo de aprendizagem em que os sujeitos assumem a Educação em Saúde como conceito de promoção da saúde (Costa, 1986; Garcia, 1986; Czeresnia et al., 1995).

Diversos órgãos internacionais recomendam a utilização de Educação em Saúde como instrumento de transformação das práticas inadequadas de saúde, seja em relação aos indivíduos como também aos próprios profissionais. Reforçando este aspecto, diversos estudiosos opinam que o grande desafio do Sistema Único de Saúde (SUS) firma-se na reorientação de novas práticas de saúde (Brasil..., 1994; Monteiro, 1995; Boog, 1997).

Face à recomendação de respeitados técnicos para implementá-la - a Educação em Saúde - no âmbito dos Serviços Públicos de Saúde, somada ao fato de que sempre esteve presente na formação dos profissionais nutricionistas e, na atualidade, inserida em programas de governo, justifica-se no momento a análise dessas práticas que norteiam as ações de saúde. Nesse sentido, a educação se atrela à comunicação em saúde como forma de acesso às informações, emergindo a saúde como direito civil; e a educação, através de diferentes processos pedagógicos, instrumentaliza esta prática. Segundo Minayo (1992) a saúde, por ser uma questão humana e existencial, é uma problemática compartilhada indistintamente por todos os segmentos sociais. Contudo, as condições sociais e de trabalho modelam formas diferenciadas de pensar e sentir a saúde. Isto a coloca para todos os grupos sociais de uma forma específica e peculiar, envolvendo uma complexa interação entre diferentes aspectos (físicos, psicológicos, culturais, sociais, ambientais). Nesse contexto a educação, enquanto forma de explicar os fenômenos, serve como balisamento para compreensão da realidade (Giddens, 1991).
Nesse contexto, Educação em Saúde pensada enquanto prática crítica e conscientizadora, vem em contra- posição à educação em saúde dantes utilizada para regulamentar, enquadrar, controlar todos os gestos, atitudes, comportamentos, hábitos e discursos das classes subalternas, além de destruir ou apropriar-se dos modos e usos do saber e de sua visão sobre o corpo, da saúde, da doença (Benjamin, 1991; Vasconcelos, 1997).

Considerando a importância do aspecto Educação em Saúde no entendimento do processo saúde/doença, na busca de mudanças de condutas e procedimentos dos indivíduos, sejam eles usuários do sistema de saúde ou mesmo profissionais da área, esta temática carece de uma apresentação de como vem sendo abordada nos Serviços; para tanto este estudo servirá como demonstração do exercício prático do processo educativo voltado para os interesses da saúde coletiva em seus diferentes campos de atuação no município de João Pessoa, Paraíba (PB).

O relato de experiências em Educação em Saúde e Nutrição nos serviços públicos de saúde são raros, muito embora esta abordagem venha merecendo o reconhecimento dos técnicos e do próprio governo como forma de intervenção na saúde da população em geral. A Educação Nutricional neste momento se distancia de um ideal de ensino para passar a preencher uma função específica de instrução a respeito de como resolver determinados problemas identificados a partir de dados epidemiológicos, envolvendo em seu conceito, aspectos de sociologia em lugar dos preceitos médicos como mentora dos programas educativos em saúde (Freitas, 1997).

Este estudo tem o objetivo de descrever as diferentes práticas de Educação em Saúde e Nutrição realizadas pelos Serviços de Saúde, de forma a propiciar aos alunos de Graduação da área de Saúde, em especial ao alunado do Curso de Nutrição, um referencial que demonstre como a temática Educação em Saúde e Nutrição está caracterizada no município de João Pessoa, PB. Pretendeu-se através deste estudo, por ocasião do cumprimento da Disciplina de Educação Nutricional, apresentar um conhecimento situacional das práticas de Educação em Saúde e Nutrição, desenvolvidas nos Serviços Médico-Assistenciais, possibilitando aos mesmos desenvolver uma visão crítica da prática do profissional de Nutrição e/ou de outra especialidade que esteja à frente deste processo de trabalho educativo.

Para atingir este propósito foram visitados os diferentes centros de saúde do município, envolvendo serviços públicos (SUS) e privados/conveniados (Não-SUS), discriminando de que forma esse processo ocorre, quais os profissionais diretamente envolvidos e quais os impactos observados. Espera-se que este estudo possibilite aos profissionais da área um conhecimento atualizado, identificando a existência de programas e/ou atividades de Educação em Saúde Nutricional nos Serviços Assistenciais, assim como uma discussão sobre o enfoque das ações desenvolvidas e, por fim, a importância, do ponto de vista dos profissionais de saúde, do processo educativo em saúde como um componente de atenção à mesma. 


\section{MATERIAL E MÉTODOS}

A pesquisa buscou alcançar o maior número de Serviços do município. Em geral, existe uma aproximação na distribuição de serviços de saúde segunda as categorias administrativas na amostra investigada, em relação ao universo de Serviços existentes no município. Foi dada preferência àqueles que recebem a maior demanda de assistência da população local.

A princípio foram catalogados todos os Serviços de Saúde do município de João Pessoa, PB em número de 70 , incluindo Hospitais, Centros de Saúde, Ambulatórios, Unidades Centrais e Unidades de Serviços Especiais, pertencentes a diferentes vínculos administrativos (SUS e Não-SUS).

Considerando a localização geográfica das Unidades de Saúde, o município foi subdivido de modo a facilitar a distribuição dos auxiliares de pesquisa por áreas com vistas a garantir a obtenção dos dados e uma cobertura total dos serviços catalogados. A equipe de auxiliares de pesquisa foi formada por quatro acadêmicos de Nutrição que receberam um treinamento prévio sobre aplicação de questionários e entrevistas em pesquisa de campo, no qual foi elaborado e testado um instrumento de coleta de informações, finalizando com um formulário que constou as indagações sobre a prática da Educação em Saúde nesses serviços. As entrevistas foram realizadas no período de janeiro a março de 1996, sob a coordenação de um professor/pesquisador, dirigidas aos profissionais de saúde que estavam presentes no momento da visita.

Não foi definido, a priori, o que seria considerado atividade de Educação em Saúde. Propositadamente foi dado aos profissionais a liberdade para relatarem as atividades realizadas pelos Serviços que no entendimento deles fossem consideradas como de Educação em Saúde. Para delimitar um espaço temporal como objeto de investigação, foi tomado como ponto de corte o período de janeiro de 1995 a março de 1996. O uso de formulário próprio de pesquisa, aplicado em cada Serviço de Saúde visou a obtenção de informações sobre a existência ou não de programas e/ou atividades educativas que foram realizadas durante o período definido e a descrição das respectivas atividades educativas, segundo a unidade assistencial, identificação do pessoal, programação, duração, população alvo, entre outras informações.

\section{RESULTADOS E DISCUSSÃO}

Participaram deste estudo 65 Unidades de Serviços de Saúde, sendo representado 93,0\% do total das Unidades catalogadas $22(33,8 \%)$ de administração municipal, 25 $(38,5 \%)$ de estadual, $12(18,5 \%)$ de federal e $6(9,2 \%)$ particulares, envolvendo 59 (91,0\%) Centros de Saúde do SUS e $6(9,0 \%)$ Não-SUS (Tabela 1$)$.
Tabela 1. Distribuição das Unidades de Saúde investigadas, segundo níveis administrativos e categorias de assistência médica, João Pessoa, PB, 1996.

\begin{tabular}{lcc}
\hline Níveis administrativos $^{*}$ & Caracterização das Unidades \\
\hline & $\mathrm{n}$ & $\%$ \\
Municipal & 22 & 33,8 \\
Estadual & 25 & 38,5 \\
Federal $^{(* *)}$ & 12 & 18,5 \\
Particular & 6 & 9,2 \\
\hline Total & $65^{* * *}$ & 100,0 \\
\hline
\end{tabular}

(*) Apenas o serviço particular não fazia parte do SUS (Não-SUS)

${ }^{* *}$ Pela complexidade do Hospital Universitário, cada Serviço Ambulatorial foi considerado como uma Unidade específica

${ }^{(* * *)}$ Do total dos Serviços catalogados, em cinco deles não foi possível realizar a pesquisa

Foi observado que 48 (74,0\%) Unidades realizam alguma atividade educativa, enquanto que 17 (26,0\%) das Unidades declararam não realizar. Apesar da constatação do número considerável de Serviços que realizam atividades de Educação em Saúde, pelas entrevistas realizadas, em sua maioria elas não tem caráter sistemático (Tabela 2).

Embora houvéssemos catalogados 11 Unidades particulares (Não-SUS), apenas 6 aceitaram participar da investigação. Nas outras, não se obteve informações por motivos alheios à pesquisa: os profissionais não aceitaram responder as perguntas da entrevista ou mesmo a responder o questionário por escrito. Entre esses Serviços é sugestivo dizer que, através da recusa de seus profissionais em aceitar serem entrevistados, há uma indicação da ausência dessas atividades nos seus procedimentos, o que os diferenciou da maioria dos outros Serviços.

\section{Envolvimento dos profissionais de saúde em atividades educativas}

Quanto ao envolvimento dos profissionais de saúde de nível superior nas atividades educativas, o estudo demonstrou que há maior participação de assistentes sociais e enfermeiras, respectivamente $22(75,8 \%)$ e 30 (75,0\%), seguido de psicólogas, 22 (73,3\%). Investigando a participação do nutricionista nessas atividades, foi verificado um envolvimento de apenas 12 (57,1\%) (Tabela 3). Apesar dessa realidade, foi observado que há possibilidade de trabalho em equipe com os profissionais de saúde em mais da metade dos Serviços, mesmo que esta não seja composta por todos os profissionais. A inserção do nutricionista nos Serviços de Saúde ainda é modesta, o que se verifica pela falta deste em mais da metade dos Serviços investigados. Considerando a oferta desse profissional no mercado de trabalho, seria ideal que houvesse um maior aproveitamento deste, em virtude de seu potencial técnico. 
Em sua maioria, os quadros de profissionais dos Serviços de Saúde, seguem a mesma tendência da participação desses nas ações de Educação em Saúde. Novamente aparece a tríade formada por assistentes sociais, enfermeiras e psicólogas. O nutricionista, que na sua formação acadêmica tem recebido um forte estímulo para atuação nas ações de Educação em Saúde, efetivamente não desenvolve em sua prática estas ações. Sua participação nos Serviços não corresponde ao investimento em sua formação. Apenas $57,1 \%$ dos nutricionistas identificados participam das ações de Educação em Saúde. Analisando sua participação nos diferentes níveis administrativos, observa-se que nos Serviços municipal e estadual sua participação ocorre em $55,5 \%$ e $28,0 \%$, respectivamente. Contudo, no nível federal sua atuação é 100,0\%; vale ressaltar que esta Unidade de Saúde refere-se ao Hospital Universitário (Hospital Escola) que tem um caráter diferenciado dos demais (Tabela 4).

Tabela 2. Distribuição das Unidades de Saúde, segundo níveis administrativos e realização de atividades educativas. João Pessoa, PB, 1996.

\begin{tabular}{|c|c|c|c|c|c|c|}
\hline \multirow{3}{*}{ Níveis administrativos } & \multicolumn{4}{|c|}{ Unidades que realizam Educação em Saúde } & \multicolumn{2}{|c|}{ Total } \\
\hline & \multicolumn{2}{|c|}{ Sim } & \multicolumn{2}{|c|}{ Não } & \multirow[b]{2}{*}{$\mathrm{n}$} & \multirow[b]{2}{*}{$\%$} \\
\hline & $\mathrm{n}$ & $\%$ & $\mathrm{n}$ & $\%$ & & \\
\hline Municipal & 18 & 37,5 & 4 & 23,5 & 22 & 34,0 \\
\hline Estadual & 17 & 35,4 & 8 & 47,0 & 25 & 38,0 \\
\hline Federal & 11 & 23,0 & 1 & 6,0 & 12 & 18,0 \\
\hline Particular & 2 & 4,1 & 4 & 23,5 & 6 & 10,0 \\
\hline Total & 48 & 100,0 & 17 & 100,0 & 65 & 100,0 \\
\hline
\end{tabular}

Tabela 3. Distribuição dos profissionais de nível superior, segundo sua participação em atividades de Educação em Saúde. João Pessoa, PB, 1996.

\begin{tabular}{|c|c|c|c|c|}
\hline \multirow{2}{*}{ Categorias profissionais } & \multicolumn{2}{|c|}{ Distribuição por Unidades } & \multicolumn{2}{|c|}{ Distribuição na participação de Educação em Saúde } \\
\hline & $\mathrm{n}$ & $\%$ & $\mathrm{n}$ & $\%$ \\
\hline Enfermeiro & 40 & 17,0 & 30 & 75,0 \\
\hline Médico & 37 & 15,0 & 20 & 54,0 \\
\hline Odontólogo & 32 & 13,0 & 10 & 31,2 \\
\hline Psicólogo & 30 & 12,5 & 22 & 73,3 \\
\hline Assistente Social & 29 & 12,0 & 22 & 75,8 \\
\hline Nutricionista & 21 & 9,0 & 12 & 57,1 \\
\hline Fisioterapia & 18 & 7,5 & 4 & 22,2 \\
\hline Administrador & 17 & 7,1 & 2 & 11,7 \\
\hline Farmacêutico & 14 & 6,0 & - & - \\
\hline Educador Física & 2 & 0,9 & 1 & 50,0 \\
\hline Total & 240 & 100,0 & 123 & - \\
\hline
\end{tabular}

Tabela 4. Distribuição das Unidades de Saúde com Serviço de Nutrição, segundo nível administrativo e participação do nutricionista nas ações de Educação em Saúde. João Pessoa, PB, 1996.

\begin{tabular}{|c|c|c|c|c|}
\hline \multirow{2}{*}{ Níveis administrativos } & \multicolumn{2}{|c|}{ Unidades de Saúde com Serviço de Nutrição } & \multicolumn{2}{|c|}{ Unidades com nutricionistas participantes das ações } \\
\hline & $\mathrm{n}$ & $\%$ & $\mathrm{n}$ & $\%$ \\
\hline Municipal & 9 & 43 & 5 & 55 \\
\hline Estadual & 7 & 34 & 2 & 28 \\
\hline Federal & 4 & 19 & 4 & 100 \\
\hline Particular & 1 & 4 & 1 & 100 \\
\hline Total & 21 & 100 & 12 & - \\
\hline
\end{tabular}


Investigando a forma de participação dos profissionais de saúde, bem como dos usuários nas ações de Educação em Saúde nos Serviços, os dados demonstraram que os profissionais de saúde atuam nas ações com maior freqüência de forma individualizada.

\section{Importância atribuída à Educação em Saúde}

Foi verificado, pelos depoimentos obtidos dos profissionais no momento da pesquisa, que todos afirmaram ser o tema Educação em Saúde um importante aspecto no que diz respeito à assistência médica, muito embora não tenham confirmado essas posições em suas práticas, o que, segundo Vasconcelos (1997), faz-se necessário para que possam adquirir sentido e sustentação. Isto fica evidenciado pelas próprias palavras de alguns entrevistados quando indagados sobre "qual a importância do aspecto educativo na questão da saúde ?" conforme os depoimentos transcritos abaixo:

"É importante para fazer com que o paciente tenha maior conhecimento da doença, tendo conseqüentemente uma qualidade de vida melhor".

(Nutricionista, Serviço Federal).

"É importante porque é um componente do tratamento do paciente, pois através das informações ele começa a entender a doença e como tratá-la".

(Enfermeira, Serviço Federal).

"A educação sanitária é primordial para o bem-estar da população. É a partir da atividade educacional que pode-se ter uma população sadia".

(Enfermeira, Serviço Municipal).

"Toda transformação social passa por uma educação em saúde; por isso a necessidade de se educar uma população".

(Nutricionista, Serviço Municipal).

"A saúde enquanto qualidade de vida, deve ser mantida no nível elevado de prevenção, e esse aspecto se dá graças à educação, veículo primordial nas questões preventivas, visto que a informação conduz o indivíduo aos conhecimentos essenciais".

(Psicóloga, Serviço Estadual).

"Dentro do contexto da saúde preventiva, onde a equipe multiprofissional deve estar envolvida, a educação comunitária é ponto de partida essencial para alcançarmos êxito no relacionamento equipe-comunidade".

(Enfermeira, Serviço Estadual).

Todos esses depoimentos sugerem que outras razões ocorrem para que estas concepções não se traduzam em práticas nos Serviços.

\section{Estratégias de ensino}

Analisando as estratégias pedagógicas utilizadas pelos profissionais de saúde nos respectivos Serviços, observou-se maior freqüência do uso de palestras (64\%), cartazes (44\%) e folhetos (40\%), em relação às demais. Ao analisar a utilização das estratégias segundo os níveis administrativos dos Serviços, verificou-se a mesma proporção entre as estratégias aplicadas, sendo que os Serviços estaduais foram os que apresentaram estratégias pedagógicas mais diversificadas. Observou-se também a existência de vários recursos audiovisuais para aplicação das ações de Educação em Saúde, apesar desta diversidade não estar presente na maioria dos serviços. Talvez, falte conhecimento para a utilização dos recursos disponíveis. O que os Serviços definem como "palestra", é qualquer abordagem verbal dirigida à população usuária, sendo esta individual ou coletiva. Até mesmo uma orientação ambulatorial entre médico e paciente foi considerada palestra e, esta, atividade de Educação em Saúde. As situações encontradas apontam para aspectos referidos por Boog (1997), que afirma que há receptividade a essas práticas, mas não ocorre possibilidade efetiva no âmbito institucional (Tabela 5).

Tabela 5. Distribuição das estratégias pedagógicas utilizadas nos Serviços de Saúde. João Pessoa, PB, 1996.

\begin{tabular}{lcc}
\hline \multirow{2}{*}{ Estratégias utilizadas } & \multicolumn{2}{c}{ Unidades de Saúde } \\
\cline { 2 - 3 } & $\mathrm{n}$ & $\%$ \\
\hline Palestras & 41 & 85,0 \\
Cartazes & 28 & 58,0 \\
Folhetos & 26 & 54,0 \\
Álbum seriado & 12 & 25,0 \\
Vídeos/TV & 9 & 19,0 \\
Slides & 4 & 8,0 \\
Transparências & 4 & 8,0 \\
Visita domiciliar & 2 & 4,0 \\
Faixas & 2 & 4,0 \\
Carro de som & 2 & 4,0 \\
Outras & 7 & 14,5 \\
\hline
\end{tabular}

(*) Considerando apenas as Unidades de Saúde que trabalham com Educação em Saúde (48)

\section{Avaliação do processo educativo}

Verificando como os Serviços de Saúde avaliam suas ações educativas, demonstrou-se que a forma de avaliação na maioria dos Serviços foi "retorno do paciente", critério este presente em 50\% dos Serviços investigados, seguido por "depoimento pessoal" (27\%). Contudo, 19\% dos Serviços não referiram utilizar nenhuma avaliação destas ações. Diante desses achados, a falta de critérios de avaliação parece sugerir que os Serviços não têm um planejamento e programação das ações de Educação em Saúde. A maioria considera que suas ações tiveram impacto positivo pelo retorno do usuário, partindo-se da premissa de que se o paciente retorna ao Serviço, foi porque as ações tiveram um bom resultado (Tabela 6). 
Tabela 6. Formas de avaliação utilizadas pelos Serviços nas ações de Educação em Saúde. João Pessoa, PB, 1996.

\begin{tabular}{lcc}
\hline \multirow{2}{*}{ Formas de avaliação } & \multicolumn{2}{c}{ Distribuição das Unidades de Saúde } \\
\cline { 2 - 3 } & $\mathrm{n}$ & $\%$ \\
\hline Retorno dos pacientes & 24 & 50 \\
Depoimento pessoal & 13 & 27 \\
Diminuição de incidências & 4 & 8 \\
Aumento da demanda & 1 & 2 \\
Visitas periódicas & 1 & 2 \\
Reunião com a equipe & 5 & 10 \\
Observação do processo & 4 & 8 \\
\hline
\end{tabular}

(*) Considerando o número de Unidades que realizam atividades de Educação em Saúde (48) e a utilização de mais de uma forma de avaliação por serviço

\section{População alvo dos programas educativos}

Verifica-se que a população alvo concentra-se em mulheres gestantes (44\%), crianças pré-escolares (42\%) e casais (40\%). Ficou indicado que os Serviços, de um modo geral, não oferecem cobertura satisfatória dos grupo-alvo principalmente àqueles considerados vulneráveis, quais sejam mulheres grávidas e crianças menores de cinco anos de idade. Cada Serviço cria programas de assistência específicos, e adota determinadas ações educativas, não fazendo uma relação direta com a realidade da própria comunidade. Isto se dá, talvez, pela falta de conhecimento prévio sobre as necessidades da população-alvo, ou porque os Serviços executam programas específicos por uma demanda gerada pelo nível central e não pela necessidade da população local (Tabela 7).

Tabela 7. Freqüência de Unidades de Saúde que desenvolvem ações educativas para os grupos-alvo. João Pessoa, PB, 1996.

\begin{tabular}{lcc}
\hline \multirow{2}{*}{ População alvo } & \multicolumn{2}{c}{ Unidades de Saúde $^{*}$} \\
\cline { 2 - 3 } & $\mathrm{n}$ & $\%$ \\
\hline Gestantes & 21 & 44 \\
Pré-escolares & 20 & 42 \\
Casais & 19 & 40 \\
Mães com neonato & 16 & 33 \\
Adolescentes & 12 & 25 \\
Idosos & 2 & 4 \\
Deficientes & 1 & 2 \\
Diabéticos & 1 & 2 \\
Não especificam & 8 & 17 \\
\hline
\end{tabular}

(*) Considerando apenas as Unidades de Saúde que trabalham com Educação em Saúde (48)

\section{Envolvimento da população}

O estudo analisou também de que forma os Serviços envolvem a população usuária. Justificou-se que na maior parte das Unidades não existe mecanismos próprios para este fim, por trabalharem simplesmente com a livre demanda (Tabela 8). Destacam-se os Serviços municipais que utilizam outros procedimentos para envolver a população usuária. Os Serviços de Saúde não dispõem de mecanismos para ir à busca dos usuários (comunidade); classicamente, espera-se que a eles venham e dessa forma ocorre também com as ações de Educação em Saúde. Este aspecto por si só limita, em muito, a abrangência das atividades educativas, o que sugere a ausência de planejamento e/ou programas que as envolvam como uma prática constante.

Tabela 8. Formas de envolvimento da população nas ações de Educação em Saúde desenvolvidas nos Serviços de Saúde. João Pessoa, PB, 1996.

\begin{tabular}{lcc}
\hline \multirow{2}{*}{ Formas de envolvimento } & \multicolumn{2}{c}{ Distribuição das Unidades de Saúde } \\
\cline { 2 - 3 } & $\mathrm{n}$ & $\%$ \\
\hline Livre demanda & 40 & 82 \\
Atendimento de solicitação & 1 & 2 \\
Voluntário & 2 & 4 \\
Visita domiciliar & 2 & 4 \\
Convite da enfermaria & 1 & 2 \\
Não específica & 3 & 6 \\
\hline
\end{tabular}

(*) Considerando apenas as Unidades de Saúde que trabalham com Educação em Saúde (48)

\section{Conteúdo dos programas educativos}

Investigando as temáticas de Educação em Saúde, verificou-se diversidade de temas, entre os quais, destacaram-se: "Vacinação" (44\%), "Planejamento familiar", "Aleitamento materno" e "Pré-natal" (37\%) e "Higiene bucal/pessoal" (34\%). Nenhum dos temas referidos está sendo abordado em pelo menos metade dos Serviços investigados. Um dado que chamou a atenção foi a diversidade de temas abordados nas ações educativas, o que é positivo, mas não se sabe se estes temas correspondem realmente às necessidades dos usuários, visto que não foi objetivo da pesquisa averiguar quais os critérios de escolha para os temas desenvolvidos (Tabela 9).

\section{Freqüência do desenvolvimento das ações educativas}

Considerando a existência da realização de ações de Educação em Saúde nos Serviços, foi verificada a freqüência com que essas ações são desenvolvidas. Esta análise revelou uma maior freqüência diária (44\%) e semanal (41\%), independentemente do nível 
administrativo. Vale ressaltar que foi computado mais de um tipo de freqüência por Serviço de Saúde investigado (Tabela 10), mas não existe uma sistemática, nem tão pouco mecanismos que garantam que estas ações sejam desenvolvidas com regularidade. Por outro lado, como o conceito do que seja Educação em Saúde é algo muito frágil, a resposta dada à pergunta sobre a freqüência com que essas ações acontecem não foram precisas. As ações educativas se dão freqüentemente junto a grupos, o que ocorre em $65 \%$ dos serviços (Tabela 11 ).

Tabela 9. Freqüência das temáticas de Educação em Saúde, nas Unidades de Saúde. João Pessoa, PB, 1996.

\begin{tabular}{lcc}
\hline \multirow{2}{*}{ Temáticas desenvolvidas } & \multicolumn{2}{c}{ Distribuição das Unidades de Saúde } \\
\cline { 2 - 3 } & $\mathrm{n}$ & $\%$ \\
\hline Vacinação & 21 & 44 \\
Aleitamento materno & 18 & 37 \\
Planejamento familiar & 18 & 37 \\
Pré-natal & 18 & 37 \\
Higiene bucal/pessoal & 16 & 34 \\
DST/AIDS & 14 & 29 \\
Prevenção do câncer & 11 & 23 \\
Educação alimentar & 10 & 21 \\
Dengue & 9 & 19 \\
Adolescência & 4 & 8 \\
Cólera & 4 & 8 \\
Tabagismo & 4 & 8 \\
Alcoolismo & 3 & 6 \\
Cuidados ao neonato & 3 & 6 \\
Reidratação oral & 3 & 6 \\
Diabetes & 2 & 4 \\
Saúde mental & 2 & 4 \\
Outras & 6 & 12 \\
\hline
\end{tabular}

(*) Considerando o número de Unidades que realizam atividades de Educação em Saúde (48) e a utilização de mais de uma forma de avaliação por serviço

Tabela 10. Formas de envolvimento da população nas ações de Educação em Saúde desenvolvidas nos Serviços de Saúde. João Pessoa, PB, 1996.

\begin{tabular}{lcc}
\hline \multirow{2}{*}{ Periodicidade } & \multicolumn{2}{c}{ Unidades de Saúde } \\
\cline { 2 - 3 } & $\mathrm{n}$ & $\%$ \\
\hline Diária & 21 & 44 \\
Semanal & 20 & 41 \\
Mensal & 13 & 27 \\
Quinzenal & 4 & 8 \\
Semestral & 4 & 8 \\
Anual & 3 & 6 \\
\hline
\end{tabular}

(*) Considerando apenas as Unidades de Saúde que trabalham com Educação em Saúde (48)
Tabela 11. Distribuição das Unidades de Saúde, segundo as formas de participação dos profissionais e usuários nas ações de Educação em Saúde. João Pessoa, PB, 1996.

\begin{tabular}{lcc}
\hline \multirow{2}{*}{ Formas de participação } & \multicolumn{2}{c}{ Distribuição } \\
\cline { 2 - 3 } & $\mathrm{n}$ & $\%$ \\
\hline Dos profissionais & 16 & 33 \\
Integrada & 32 & 67 \\
Individual & $\mathbf{4 8}$ & $\mathbf{1 0 0}$ \\
Total & & \\
Dos usuários & 31 & 65 \\
Grupal & 17 & 35 \\
Individual & $\mathbf{4 8}$ & $\mathbf{1 0 0}$ \\
\hline Total & & \\
\hline
\end{tabular}

\section{CONCLUSÃO}

Os Serviços Médico-Assistenciais de Saúde que respondem pelo atendimento da maioria da população do município de João Pessoa, concentram-se em Centros de Saúde e Hospitais. As categorias profissionais numericamente mais representadas são médicos e enfermeiros. No entanto, as ações de Educação em Saúde se dão principalmente através de assistentes sociais, enfermeiros e psicólogos, apesar de existirem também outros profissionais.

Mesmo que a pesquisa tenha resgatado diversas estratégias pedagógicas que são empregadas nos Serviços, palestras, cartazes e folhetos são os mais encontrados. Apesar de não existir critérios objetivos de avaliação das ações de Educação em Saúde, a maioria dos Serviços considera que o "retorno do paciente" às ações é a forma de avaliação mais empregada.

Quanto à existência de Serviço de Nutrição nas Unidades de Saúde investigadas, verificou-se que existe um maior número de unidades sem esse, e quando há, a sua participação em ações educativas é pequena, a considerar os serviços municipais e estaduais, com 55\% e $28 \%$ de participação, respectivamente.

A clientela das ações educativas está concentrada entre gestantes, pré-escolares e casais. As temáticas apontadas com maior freqüência pelos profissionais foram: aleitamento materno, doenças sexualmente transmissíveis/ Sindrome de Imunodeficiência Adquirida, planejamento familiar, pré-natal, vacinação, higiene bucal/pessoal, dentre as 25 temáticas relatadas. Estas ações são oferecidas à livre demanda de clientes que buscam os Serviços, sendo desenvolvidas diária e semanalmente.

Diante do exposto e de forma a contribuir para a busca de uma assistência mais efetiva, apresentamos algumas recomendações que consideramos oportunas para o bom desenvolvimento das ações de saúde: 
- No nível central a exemplo da Secretaria Municipal de Saúde - discuti propostas de institucionalização das ações de uma política de Educação em Saúde, com o envolvimento dos profissionais e da população;

- Adotar como rotina dos serviços as ações de Educação em Saúde, diferenciando-se assim da ação isolada de um profissional;

- Oferecer aos profissionais capacitação para abordagem de práticas em Educação em Saúde;

- Possibilitar a participação da comunidade nas discussões sobre as ações educativas;

- Promover oportunidades e recursos para os profissionais desenvolverem ações de educativas.

\section{REFERÊNCIAS BIBLIOGRÁFICAS}

BENJAMIN, F.S. Educação e medicina social: uma tentativa crítica. São Paulo : Cortez, 1991. p.108.

BEZERRA, A. Conversando com os agentes. Cadernos de Educação Popular, Rio de Janeiro, n.3, p.9-31, 1986.

BOOG, M.C.F. Educação nutricional: passado presente, futuro. Revista de Nutrição da PUCCAMP, Campinas, v.10, n.1, p.5-19, 1997.

BRASIL. Ministério da Saúde. A lei orgânica de Saúde. Brasília, 1990. p.88.

BRASIL. Ministério da Saúde. Secretaria Executiva. Coordenação Geral de Desenvolvimento de Recursos Humanos Para o SUS. Capacitação pedagógica para Instrutores/Supervisores na área de saúde. Brasília, 1994. 60p.
COSTA, N.R. Estado, educação e saúde: a higiene da vida cotidiana. Educação e Saúde, São Paulo, n.4, p.5-27, 1986. (Centro de Estudos Educação e Sociedade).

CZERESNIA, D., SANTOS, E.M., BARBOSA, R.H.S. MONTEIRO, S. AIDS: pesquisa social e educação. São Paulo : Hucitec, 1995. 206p.

FREITAS, M.C.S. Educação nutricional: aspectos sócio-culturais. Revista de Nutrição da PUCCAMP, Campinas, v.10, n.1, p.45-49, 1997.

GARCIA, P.B. Saber popular/Educação popular. Cadernos de Educação Popular, Rio de Janeiro, n.3, p.33-62, 1986.

GIDDENS, A. As conseqüências da modernidade. São Paulo : [s.n.], 1991. p.179.

LAVINSKY, L. Saúde: informações básicas. Porto Alegre : Editora Universitária UFRGS, 1988. 191p.

MINAYO, M.C.S. O desafio do conhecimento: pesquisa qualitativa em saúde. São Paulo : Hucitec, 1992. p.269.

MONTEIRO, C.A. Velhos e novos males da saúde no Brasil: a evolução do país e de suas doenças. São Paulo: Hucitec, 1995. 359p.

PITTA, A.M.R. A comunicação. Serviço de Saúde. Populações: modelos explicativos e desafios a partir de discussões recentes. Rio de Janeiro, 1994. p.126. Dissertação (Mestrado em Saúde Coletiva)- Instituto de Medicina Social, Universidade Federal do Rio de Janeiro, 1994.

VALENTE, F.L.S. Em busca de uma educação nutricional crítica. In: Fome e desnutrição, determinantes sociais. São Paulo : Cortez, 1986. p.56-64.

VASCONCELOS, E.M. Educação popular nos Serviços de Saúde. São Paulo : Hucitec, 1997. p.167.

Recebido para publicação em 1 de agosto de 1997 e aceito em 28 de abril de 1999. 